\title{
ИСПОЛЬЗОВАНИЕ ЭКОНОМИКО-МАТЕМАТИЧЕСКИХ МОДЕЛЕЙ В УСЛОВИЯХ ЦИФРОВОЙ ТРАНСФОРМАЦИИ ЭКОНОМИЧЕСКИХ ОТНОШЕНИЙ
}

\author{
(c) 2020 Магомадов Эмин Мухадинович \\ кандидат экономических наук, доцент кафедры учета, анализа и аудита в цифровой экономике \\ Чеченский государственный университет, Россия, Грозный \\ E-mail: chgu@yandex.ru
}

\author{
(c) 2020 Муртазалиева Амина Хамзатовна \\ 3 курс Института экономики и финансов \\ Чеченский государственный университет, Россия, Грозный
}

В статье рассмотрены экономико-математические модели динамики экономического развития предприятий, находящихся в условиях цифровой трансформации, представляющей собой процесс интеграции цифровых технологий во все аспекты деятельности предприятия и вносящей коренные изменения в технологии, операции и способы создания новой продукции.

Ключевые слова: смарт-предприятия, цифровые технологии, макроэкономическая производственная функция, цифровая трансформация.

Целью данной статьи является изучение мирового опыта в экономико-математическом моделировании в смарт-предприятиях в условиях цифровой трансформации экономических отношений.

В 2011 году на Ганноверской выставкеярмарке группой германских исследователей, бизнесменов и общественных деятелей, входящих в научно-исследовательский альянс по разработке стратегических принципов высокотехнологичного производства, был предложен термин «Промышленность 4.0» и ее принципы. Это событие ознаменовало осмысление и начало перехода к новой индустриальной революции, основанной на повсеместном применении «умных» технологий, способных полностью исключить человека из процесса принятия рутинных решений в сфере производства. Распространение информационных технологий, тотальная автоматизация самых разнообразных процессов, открытие принципиально новых материалов и безотходных способов их использования, успехи в создании кибер-физических систем, обладающих искусственным интеллектом - все это стало предпосылками для осознания новых, революционных возможностей в организации промышленного производства. Переход к новой смарт-системе производства и мероприятия по трансформации производственных отношений должны тщательно обосновываться, а наиболее эффективным инструментом описания проектируемых систем и процессов является инструментарий экономико-математического моделирования, позволяющий проводить эксперименты с проектируемой системой, изучать ее свойства, оценивать эффективность, предвосхищать возникновение проблем и ошибок без риска понести колоссальные потери, неизбежные в случае проведения экспериментов с реальной системой. Аппарат экономикоматематического моделирования в настоящее время достаточно хорошо разработан для описания любых, даже самых сложных процессов и систем, однако, новизна решаемых задач при создании смарт-предприятий не позволяет сделать однозначный выбор в пользу применения каких-то определенных инструментов, в связи с чем целесообразно изучение зарубежного опыта применения этих методов при создании смартпредприятий, поскольку у развитых стран в данном вопросе уже есть определенные эмпирические знания. Поэтому целью данной статьи является изучение мирового опыта в использовании экономико-математических моделей на предприятиях в условиях цифровой трансформации экономических отношений.

Одним из важных направлений формирования цифровой экономики является организация процессов цифровизации производственных предприятий. Эти процессы сопровождаются широким внедрением таких новых технологий, как обработка и аналитика больших данных, 
машинное обучение, искусственный интеллект, роботизация, дополненная реальность, промышленный интернет вещей (IoT), 3D-печать, облачные вычисления и т.д. Широкое развитие и диффузия цифровизации стали возможными за счет снижения стоимости технологий, увеличения вычислительных мощностей и скорости передачи данных. Цифровая трансформация предприятия опирается на операционную цифровизацию, представляющую собой внедрение цифровых инструментов для повышения эффективности предприятия. Внедрение цифровых инструментов в операционную деятельность позволяет предприятиям удаленно управлять физическими элементами оборудования предприятия и его производством в целом. Набор эффективных экономико-математических методов моделирования представляет для «умного предприятия» инструментарий, позволяющий увеличивать выпуск готовой продукции, снижать издержки, сокращать расход материалов, повышать доступность оборудования. Как отмечается в исследовании исполнительного комитета СНГ по состоянию, проблемам и перспективам развития информационного общества, необходимо разрабатывать новые методы, позволяющие правильно понять и исследовать открывающуюся перед ним новую высокодинамичную информационную картину мира. Методы экономикоматематического моделирования, позволяющие получить объективные и непредвзятые количественные обоснования, должны занять среди этих методов важную нишу. Исходя из приведенного анализа актуальных направлений исследования становления смарт-промышленности можно выделить следующие перспективные направления экономико-математического моделирования смарт-предприятий:

1. В первую очередь интерес вызывает эволюция макроэкономической производственной функции в связи с переходом к неоиндустриальной смарт-экономике. Использование методов экономико-математического моделирования дает возможность теоретически обосновать качественные изменения этой функции в связи с появлением новых технологических комбинаций классических факторов производства, и возможным появлением нового фактора производства в виде информатизации или искусственного интеллекта.

Можно предложить несколько спецификаций производственной функции предприятий с учетом действия этого нового фактора (обозначим его I):

- мультипликативная функция (аналог Кобба-Дугласа):

$$
y=a_{0} K^{a_{1}} L^{a_{2}} I^{a_{3}} .
$$

где факторы производства представлены в натуральном измерении;

- аддитивно-мультипликативная функция:

$y=a_{1} K+a_{2} L+a_{3} I+a_{4} K L+a_{5} K I+a_{6} L I$

где факторы производства представлены в стандартизированном виде.

Второй вариант может оказаться более информативным для статических моделей, поскольку способен отразить различные мультипликативные эффекты, получаемые от разных сочетаний факторов. Если же рассматривать развитие производственной функции в динамике, то информативнее может оказаться первый вариант, так как есть основания полагать, что параметр $a_{3}$ описывается некой $S$-образной кривой, зависящей от времени, например кривой Гомперца или логистической кривой:

$$
a_{3}=\frac{1}{1+b e^{a_{1}}} .
$$

Выбор $S$-образной кривой обусловлен лавинообразным характером процессов информатизации, и, возможно, развития искусственного интеллекта, когда приросты зависят от достигнутого уровня, и сначала идут с ускорением развития, а затем по мере насыщения - с замедлением.

2. Всевозможные вариации модели Леонтьева «Затраты-Выпуск» и межотраслевого баланса, с помощью которых можно решать минимум три задачи:

- сквозного планирования и управления промышленностью на основе единого цифрового пространства промышленности;

- обоснования предприятий, требующих первоочередной цифровой интеграции, оценки потерь от сохранения «несмартизированных» участников создания цепочек стоимости и т.п.;

- повышения спроса в условиях внедрения цифровых бизнес-моделей и расширения цифрового взаимодействия с клиентами за счет снижения транзакционных издержек.

В качестве коэффициентов технологической матрицы модели «Затраты - Выпуск» можно использовать не только натуральное выраже- 
ние затрат, но денежное. При этом, в денежном выражении коэффициента затрат можно выделить определенные стоимостные компоненты, например, затраты труда $\left(l_{i j}\right)$, затраты на транспортировку $\left(t r_{i j}\right)$, транзакционные издержки, связанные с промежуточным и конечным потреблением продукции $\left(z_{i j}\right)$. Таким же образом можно рассматривать фактор времени $\left(t_{i j}\right)$, как элемент затрат, связанный с организацией цепочки создания стоимости. Это открывает целый пласт уже оптимизационных задач, позволяющих определить взаимосвязанные отрасли и потребителей, в наибольшей степени нуждающихся в интеграции на основании единого цифрового пространства промышленности. Рассмотрим один из вариантов общей математической постановки таких задач. Предположим, что затраты $z_{i j}$ матрицы затрат межотраслевого баланса можно снизить за счет смартизации производств в отраслях $i$ и $j$ :

$$
Z_{i j}^{\prime}=Z_{i j}\left(1-S_{i} S_{j}\right)
$$

где $S_{i} S_{j}-$ некий уровень смартизации предприятий, измеряемый величиной в диапазоне $(0 ; 1)$. (Причем, заметим, если одна из взаимодействующих сторон не является смартпредприятием, эффекта снижения затрат наблюдаться не будет). Сам же уровень смартизации предприятий является некоторой $S$-образной функцией от инвестиционных затрат К, связанных с преобразованием традиционного предприятия в смарт-предприятие:

$$
S_{i}=\frac{1}{1+b_{i} e^{-m_{i} K_{i}}}, \quad S_{j}=\frac{1}{1+b_{j} e^{-m_{j} K_{j}}} .
$$

Есть основания предполагать, что в рамках одной отрасли связь инвестиционных затрат с уровнем смартизации описывается одной и той же функцией (параметр $b$ одинаков), и отличается лишь параметром масштаба производства $\left(m_{i} m_{j}\right)$, так как очевидно, чем крупнее предприятие, тем больше «умного» оборудования необходимо внедрить, чтобы достичь того же уровня смартизации производства. Тогда задачу по снижению издержек производства за счет внедрения смарт-индустриализации в рамках ограниченных инвестиционных ресурсов можно представить в виде:

$$
\sum_{i} x_{i} \sum_{j} Z_{i j}^{\prime} \Rightarrow \min
$$

$$
\begin{aligned}
& X=(E-A)^{-1} Y, \\
& Z_{i j}^{\prime}=Z_{i j}\left(1-\frac{1}{1+b_{i} e^{-m_{i} K_{i}}} \cdot \frac{1}{1+b_{j} e^{-m_{j} K_{j}}}\right) \\
& \sum_{A_{i}} K_{i} \leq K_{\text {lim }}
\end{aligned}
$$

где $A=\left(a_{i j}\right) n \times n-$ технологическая матрица, элементы которой $a_{i j}=x_{i j} / X_{j}$ показывают, сколько продукции отрасли $i$ необходимо затратить для производства одной единицы продукции отрасли $j, Y_{n \times 1}-$ вектор-столбец конечной продукции.

3. Третьим направлением экономикоматематического моделирования смартпредприятий можно назвать вариации сетевых моделей, транспортной задачи, задачи о назначениях и т.п. Построение сетевого графа взаимодействий потребителей, производителей и других контрагентов, например, в какой-то конкретной отрасли позволит найти решение следующих проблем:

- обоснования сетевых эффектов при создании смарт-предприятий в данной отрасли и оценить тот, минимально необходимый уровень цифровизации сети, при котором затраты от дальнейшего внедрения «умных» технологий будут компенсироваться ростом эффективности сети в целом;

- в рамках ограничений на объем доступных инвестиционных ресурсов определение предприятий, нуждающихся в первоочередной цифровизации своих производств так, чтобы путь прохождения заказа от заявки до его получения оказался с минимальными издержками;

- оптимизации движения товаров (от их проектирования до потребления конечными клиентами) в условиях интернета вещей и «умной» инфраструктуры.

Стандартная целевая функция в таких задачах состоит в минимизации издержек при движении из начальной в конечную вершину.

$$
Z=\sum_{i} \sum_{J} c_{i j} x_{i j} \rightarrow \min ,
$$

где $x_{i j}$ - искомый объем груза, перемещаемый из $i$-й вершины в $j$-ю; затраты $c_{i j}$ на это перемещение (для различных дуг могут быть как постоянными, так и зависеть от объема перемещаемого груза). 
Стандартные ограничения: все потребители должны быть удовлетворены, суммарное производство равно суммарному потреблению:

$$
\begin{aligned}
& \sum_{i} x_{i j}=a_{i}, \\
& \sum_{j} x_{i j}=b_{j}, \\
& \sum_{i} a_{i}=\sum_{j} b_{j} .
\end{aligned}
$$

Очевидным расширением этой задачи является определение эффективного пути в условиях возможности смартизации отдельных предприятий, входящих в данную сеть.

Новая промышленная революция основывается на достижениях шестого технологического уклада, который характеризуется массовым внедрением технологий аддитивного производства, нанотехнологий и биоинженерии, полной цифровизацией производств, эксплуатацией киберфизических систем, обладающих искусственным интеллектом, созданием глобальной информационной сети товаров, транспорта, зданий, производств, способных взаимодействовать друг с другом самостоятельно без вмешательства человека. Страны бывшего СССР, промышленность которых основана на технологиях 3-го и 4-го технологических укладов, сильно отстают в своем развитии от западных стран, и возможности эволюционно догнать их выглядят сомнительно. В то же время создание новых предприятий, эксплуатирующих технологии 6-го уклада, может помочь им занять определенные ниши в мировом цифровом производстве. Наиболее эффективным способом обоснования экономической целесообразности создания смарт-предприятий и преобразования существующих предприятий в смарт-предприятия является использование инструментария экономико-математического моделирования, позволяющего проводить эксперименты с проектируемой системой, изучать ее свойства, оценивать эффективность, предвосхищать возникновение проблем и ошибок. Среди перспективных направлений экономикоматематического моделирования смарт-предприятий в странах с эмерджентной экономикой выделены следующие:

1) использование модификаций производственных функций - для обоснования качественных изменений в факторах производства, появления новых факторов производства, их новых технологических комбинаций;

2) применение модификаций моделей Леонтьева «затраты-выпуск» и оптимизационных моделей - для сквозного планирования и управления промышленностью, обоснования предприятий, требующих первоочередной цифровой интеграции, сокращения транзакционных издержек в условиях внедрения цифровых бизнес-моделей и расширения цифрового взаимодействия с клиентами;

3) использование модификаций сетевых моделей и моделей оптимизации - для оптимизации движения товаров (от их проектирования до потребления конечными клиентами) в условиях интернета вещей и «умной» инфраструктуры, а также для обоснования первоочередных претендентов на цифровизацию в условиях ограничений на объем доступных инвестиционных ресурсов;

4) разработка корреляционно-регрессионных моделей - для оценки экономических стохастических зависимостей, а также имитационных моделей - для оценки последствий тех или иных сценариев проведения смарт-индустриализации для занятости, доходов населения и экономики в целом.

Конкретизация постановки данных моделей и подходов к их реализации требует дополнительного углубленного изучения специфики решаемых задач и формализации отдельных институциональных факторов.

\section{Библиографический список}

1. Василенко В. (2013) Технологические уклады в контексте стремления экономических систем к идеальности. Тернополь. Т. 8. № 1. С. 65-72.

2. Воронин А.В. (2013) Моделирование технических систем. Томск: Томский политехнический университет.

3. Глазьев С. (2017) Битва за лидерство в XXI веке. Россия-США-Китай. Семь вариантов обозримого будущего. М.: Книжный мир. 
4. Евразийская экономическая комиссия (2017) Анализ мирового опыта развития промышленности и подходов к цифровой трансформации промышленности государств-членов Евразийского Экономического Союза: информационно-аналитический отчет. М.: Евразийская экономическая комиссия.

5. Каплан А.В., Каплан В.Е., Мащенко М.В., Овечкина Е.В. (2007) Решение оптимизационных задач в экономике. М.: Феникс.

6. Куприяновский В.П., Намиот Д. Е., Синягов С. А. (2016) Кибер-физические системы как основа цифровой экономики // InternationalJournalofOpenInformationTechnologies. Vol. 4. № 2. P. 18-25.

7. Куприяновский В.П., Синягов С. А., Намиот Д. Е., Уткин Н. А., Николаев Д. Е., Добрынин А. П. (2017) Трансформация промышленности в цифровой экономике - проектирование и производство // InternationalJournalo fOpenInformationTechnologies. Vol. 5. № 1. P. 50-70.

8. Maдых A.А., Охтень А. А. (2018) Моделирование трансформации влияния производственных факторов на экономику в процессе становления смарт-промышленности. Экономика промышленности. № 4 (84). С. 26-41. doi: http://doi.org/10.15407/econindustry 2018.04.026

9. Международный банк реконструкции и развития / Всемирный банк (2016) Доклад о мировом развитии «Цифровые дивиденды». Washington: Международный банк реконструкции и развития / Всемирный банк.

10. Паклин Н.Б., Орешков В. И. (2009) Бизнес-аналитика: от данных к знаниям. СПб.: Питер.

11. Трофимова Е. (2016) Киберуязвимость умных производств // ControlEngineering Россия. № 1 (61). С. 34-36.

12. Atzori L., Lera A., Morabito G. (2010) The internet of things: a survey // Computer Networks. № 54. P. $2787-2805$. DOI: 10.1016/j.comnet.2010.05.010.

13. Reimann M., Ruckriegel C. (2017) Road2CPS Priorities and Recommendations for Research and Innovation in Cyber-Physical Systems. Stuttgart: Steinbeis-Editions.

14. Yin S., Kaynak O. (2015) Big Data for Modern Industry: Challenges and Trends //Proceedings of the IEEE. Vol. 103. № 2. P. 143-146.

15. Zaitsev D.A. (2012) Universal Petri Net //Cybernetics and Systems Analysis. Vol. 48. Issue 4.P. 498511. 